\title{
Single Supply CMOS Up Level Shifter for Dual Voltage System
}

\author{
José C. García, and Juan A. Montiel-Nelson \\ University of Las Palmas de Gran Canaria \\ Las Palmas de Gran Canaria, Spain \\ Email: \{jcgarcia, montiel $\} @$ iuma.ulpgc.es
}

\author{
Saeid Nooshabadi \\ Department of Electrical and Computer Engineering \\ Michigan Technological University, USA \\ Email: saeid@mtu.edu
}

\begin{abstract}
A single supply CMOS up level shifter (rsc-ls), for upconverting signals from $0.45 \mathrm{~V}-0.9 \mathrm{~V}$ logic level range up to $1 \mathrm{~V}$ power supply voltage domain (Vdd), is introduced. From simulations results, the proposed shifter provides improvements of $63 \%$ and $69 \%$ in energy consumption and speed, respectively, when compared with a similar design, in $65 \mathrm{~nm}$ CMOS process and low threshold voltage. For a Vdd of $1.2 \mathrm{~V}$ we obtain up to $88 \%$ improvement in delay-energy product, when the input signal high voltage level is $0.7 \mathrm{~V}$ with a frequency of $500 \mathrm{MHz}$. We report a total static current reduction of up to 110 times. For an up shift of $0.45 \mathrm{~V}$ to $1.2 \mathrm{~V}$ at an output loading of $800 \mathrm{fF}$, our design offers a delay of $0.38 \mathrm{n}$ s and an energy consumption of $1.57 \mathrm{pJ}$.
\end{abstract}

\section{INTRODUCTION}

To reduce power dissipation, modern systems support multisupply voltage domains. Each domain operates at a particular power supply voltage depending on its own performance requirement. It may be even desirable to significantly reduce the power consumption by operating part of the system in subthreshold regime. A level converters are required to up and down shift the signals from/to the lower to the higher/lower power supply domains. High power efficiency is a challenge in the design of up level shifters.

A general up level converter consists of three parts; the low voltage stage (Vddl domain), a fast up converter interface circuitry, and a high voltage stage (Vdd domain), with a high drive circuitry.

One challenge in the design of up converters is the elimination or significant reduction of the undesirable static current, and therefore energy consumption. This current is the result of the high input logic (Vddl) not being high enough to completely turn off the following PMOS pull-up network that is connected to Vddh. Other challenge is the minimization of the delay in the level converters.

A subthreshold level shifter which uses a self-controlled current limiter by detecting the output error and realizes a voltage conversion from 0.1 to $1.2 \mathrm{~V}$ with limited static power consumption is presented in [1]. At $0.2 \mathrm{~V}$, the circuit operates at a maximum input frequency of $254 \mathrm{kHz}$. This circuit is useful to prolong operational lifetime from constrained power supplies for biological systems, and wireless sensors.

Typical techniques to reduce the short circuit current make use of diode-connected transistors and multi-threshold CMOS voltage (MTCMOS) to up convert the voltage signals. The cross coupled circuit topology proposed in [3] exploits the leakage current mirror technique to reduce the static current across a wide range of conversion voltages.

A Pseudo-Diode-Mirrored (PDM) level shifter with a small DC current is shown in [4] which enables resistive RAM (ReRAM) to achieve sub- $0.5 \mathrm{~V}$ read/write operations. This is useful for applications that require low power supply operations and non-volatile memory for power reduction.

The work in [5] proposed a hybrid structure comprising a modified Wilson current mirror and generic CMOS logic gates for upward and downward level conversion in a $65 \mathrm{~nm}$ technology. Bidirectional level conversion improves the energy efficiency of the interface for dynamic voltage scaling (DVS). It also helps to reduce the high amount of quiescent current that occurs when the input voltage is in subthreshold region. This region of operation limits the use of the conventional current mirror level shifter. This circuit also has the desirable property of low sensitivity to transistor sizing, threshold voltage variation, and other process variations.

In order to reduce the power area cost, as well as extending the operating range, a cross-coupled half latch structure is presented in [6]. Two off-biased PMOS transistors are inserted to provide sufficient leakage current for the proper operation the converter since the NMOS transistors in the pull-down network are able to sink that leakage current. Fig. 1, presents a modified version of the circuit in [6] where two diodes (onbiased NMOS transistors), D 0 and D1, replace two off-biased PMOS transistors. The use of low threshold voltage NMOS transistor provides a basis for a fair comparison with our work in this paper, where the key idea is to obtain a fast and low energy level converter.

The rest of this paper is organized as follows. Section II introduces the proposed voltage up level shifter $(r s c-l s)$. In Section III, simulation results are discussed and evaluated. Section IV considers post-layout simulation results of our design verifying the efficiency of the proposed circuit. Finally, this work offers a conclusion in Section VI.

\section{The Single Supply up LeVel ShIfTER Circuit TOPOLOGY}

The proposed circuit is a structure comprising three inverters and some additional transistors to reduce the static current using the logic level for node 3, as shown in Fig. 2. When 


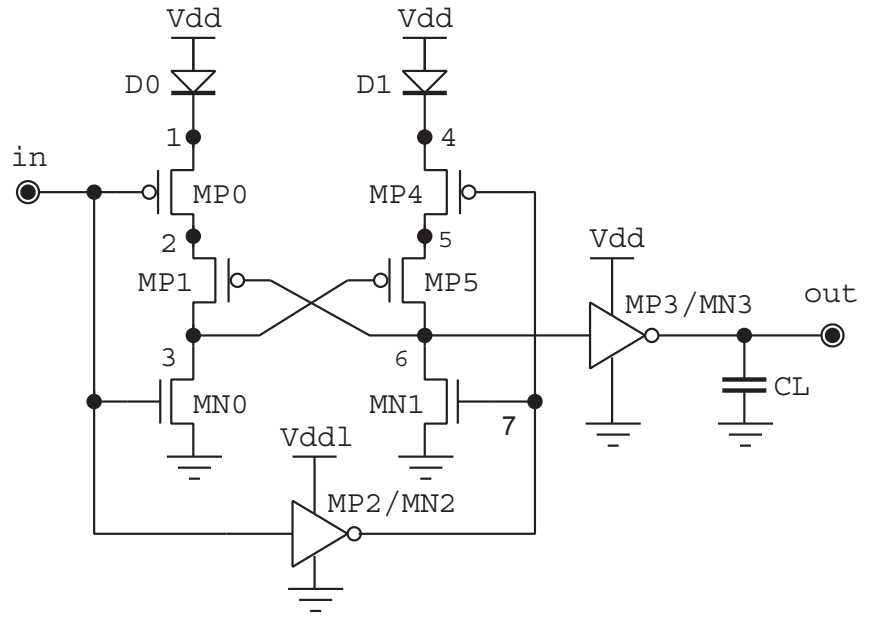

Fig. 1. The circuit diagram of $b m-l s$

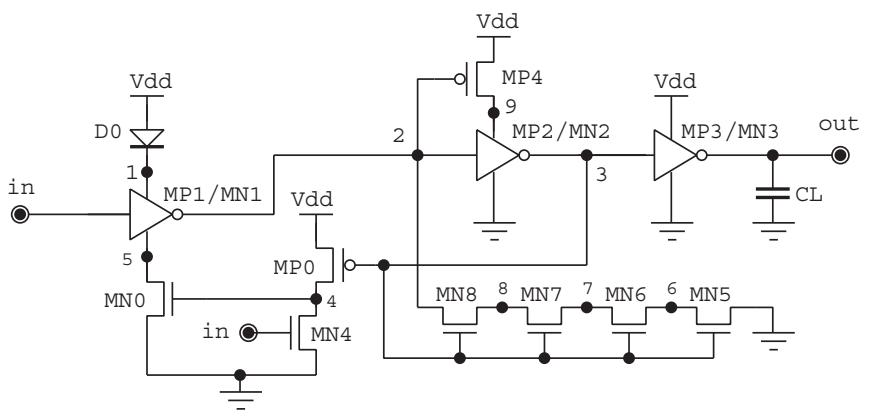

Fig. 2. Schematic for $r s c-l s$ up level shifter

node in is high, inverter MP $1 /$ MN1 produces a low level at node 2, pushing node 3 to a high level. The inverting buffer (MP 3 /MN3), designed to assure adequate output driving strength, provides a low level on node out and capacitive load CL is discharged through MN3 transistor. The NMOS diode (DO) acts as current limiter, and is used to mitigate the current contention at the beginning of the discharge transition at nodes 2 and 5. Inverter MP2/MN2 also drives the gate of MPO causing it to turn off, while the string of NMOS transistors (MN5-MN8) is turned on, which is used as intermediate stage to reduce the short circuit current and stand-by energy consumption in combination with MP $0, \mathrm{MN}$, and MN 4 transistors. The low level on node 2 is achieved through the chain of transistors (MN5-MN8). On the other hand, transistor MN 4 is turned on, pulling node 4 low, and turning MNO off. Note that the only path for the static current is through high resistance path, consisting of the chain of MN5MN8 transistors, to ground instead of current flowing out of node 5 through MNO. In this way, the short circuit current in the input inverter (MP1/MN1) is significantly reduced with respect to $b m-l s$, while the output switching speed is also improved.

When the input signal on node in is low, the voltage on node 2 is high (Vdd-VD0) and, consequently node 3 is switched to low. Moreover, node out pulls up and CL is quickly charged. This operation is also favored by current-
TABLE I

Channel Widths For Transistors IN FIg. 1, AND FIg. 2

\begin{tabular}{|c|c|c|}
\hline \multicolumn{3}{|c|}{ Fig. 1 (Active area $=310.7 \mu \mathrm{m}^{2}$ ) } \\
\hline Transistor(s) & Type & Width $(\mu \mathrm{m})$ \\
\hline $\mathrm{MP} 0$ and MP 4 & $\mathrm{P}$ & $150 \times 10.0$ \\
\hline MP 1 and MP 5 & $\mathrm{P}$ & $3 \times 10.0$ \\
\hline MP2 & $\mathrm{P}$ & $80 \times 10.0$ \\
\hline MP 3 & $\mathrm{P}$ & $1 \times 10.0$ \\
\hline MN0 and MN1 & $\mathrm{N}$ & $1 \times 10.0$ \\
\hline MN2 & $\mathrm{N}$ & $8 \times 10.0$ \\
\hline MN3 & $\mathrm{N}$ & $1 \times 10.0$ \\
\hline D0 and D1 & $\mathrm{N}$ & $40 \times 10.0$ \\
\hline \multicolumn{3}{|c|}{ Fig. 2 (Active area $=3.94 \mu \mathrm{m}^{2}$ ) } \\
\hline Transistor(s) & Type & Width $(\mu \mathrm{m})$ \\
\hline MPO & $\mathrm{P}$ & $1 \times 0.25$ \\
\hline MP 1 & $\mathrm{P}$ & $1 \times 6.0$ \\
\hline MP2 & $\mathrm{P}$ & $1 \times 2.5$ \\
\hline MP 3 & $\mathrm{P}$ & $1 \times 10.0$ \\
\hline MP 4 & $\mathrm{P}$ & $1 \times 0.65$ \\
\hline MNO & $\mathrm{N}$ & $1 \times 6.0$ \\
\hline MN1 and MN3 & $\mathrm{N}$ & $1 \times 10.0$ \\
\hline MN2 & $\mathrm{N}$ & $1 \times 4.0$ \\
\hline MN4 up to MN8 & $\mathrm{N}$ & $1 \times 0.25$ \\
\hline D0 & $\mathrm{N}$ & $1 \times 10.0$ \\
\hline
\end{tabular}

$\mathrm{Vthn}=0.28 \mathrm{~V}$, and $\mathrm{Vthp}=-0.2 \mathrm{~V}$

limiting action of the string of transistors MN5-MN8, device MP 0 , and by the presence of transistors MNO, and MN 4 which keep node 2 at a high level. In this way, transistors MN5-MN8, and MN 4 are turned off, while transistors MNO, and MNO are switched on. Node 5 is set to ground.

We used only low threshold devices to evaluate the performance of both circuit topologies. For the sake of a fair comparison, we replicated the both designs in CMOS process, while imposing a transistor length of $65 \mathrm{~nm}$ and maintaining the same ratios of $\mathrm{Wp} / \mathrm{Wn}(16 / 4)$ for the five input inverters in connected series that derive the node in for both $r s c-l s$ and $b m-l s$ circuits, and for the output inverter attached to node out. The circuits were sized as reported in Table I. The total equivalent length for the string of serially connected transistors (MN5-MN8) is $0.26 \mu \mathrm{m}$, with all transistors in the chain having the same sizes as the rest of devices in the proposed up converter. With this sizing configuration, the string draws lower static current when is turned on. Furthermore, $r s c-l s$ requires only $3.94 \mu \mathrm{m}^{2}$ of active area, or 78 times less than the respective areas for $b m-l s$ in $65 \mathrm{~nm}$ CMOS technology.

Fig. 3 illustrates the output voltage waveforms for both $b m-l s$ and $r s c-l s$, respectively. Simulations were performed considering a input signal frequency of $500 \mathrm{MHz}$, input signal voltage of $0.7 \mathrm{~V}$, and $800 \mathrm{fF}$ of capacitive load. Note that $r s c-$ $l s$ has a significantly faster response with respect to that of $b m-l s$. Our up level converter operates correctly for Vddl as low as $0.45 \mathrm{~V}$. However, $b m-l s$ fails to operate correctly when $\mathrm{Vddl}<0.7 \mathrm{~V}$. This is because the strength of the pull-down transistors is limited by $\mathrm{Vddl}$.

\section{Performance Analysis}

Pre-layout simulations were performed for both circuits, using an input signal frequency of $500 \mathrm{MHz}, \mathrm{V} d d$ fixed to $1.2 \mathrm{~V}$, 


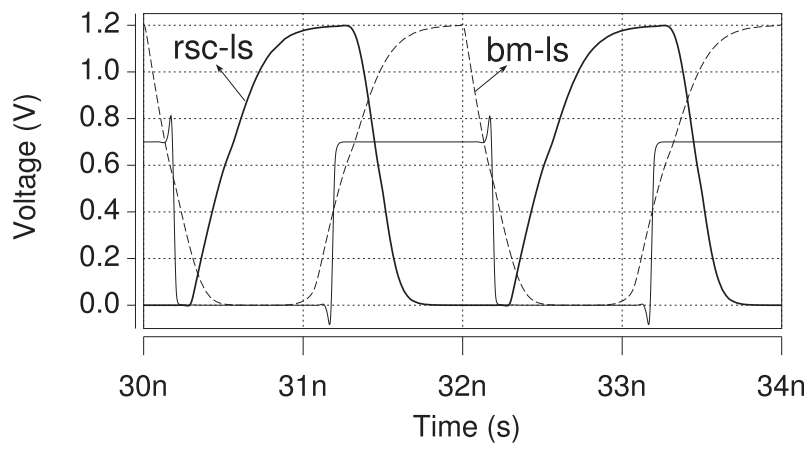

Fig. 3. Simulated pre-layout waveforms for $b m-l s$, and $r s c-l s$. A $800 \mathrm{fF}$ load capacitance is attached to the output

a parasitic capacitor of $25 \mathrm{fF}$ added to the input of each of the five inverters connected in in series that drive the node in, and an output buffer connected to the node out which is also loaded by an additional parasitic capacitor of $45 \mathrm{fF}$ in parallel to $\mathrm{CL}$. Under similar conditions, $r s c-l s$ has a $69 \%$ lower delay compared to $b m-l s$ when loaded with a load capacitance CL of $800 \mathrm{fF}$.

Our up level converter exhibits a significantly reduced energy, due to use of string of devices MN5-MN8, and devices MPO, MNO, and MN4. The efficiency of $r s c-l s$, in terms of energy consumption, is $63 \%$ higher than $\mathrm{bm}-l \mathrm{~s}$. The energydelay product for a load of $800 \mathrm{fF}$, for $r s c-l s$ is $88 \%$ lower than that for $b m-l s$.

The important feature of the proposed design is to reduce the static current. $r s c-l s$ has a 110 -fold lower current consumption $(12.18 \mu \mathrm{A} / 0.11 \mu \mathrm{A})$ than $b m-l s$ in the range of $80 \mathrm{fF}$ to $800 \mathrm{fF}$ CL load, for a $0.7 \mathrm{~V}$ input voltage.

\section{Post-LAYOUT ANALYSIS FOR $r s c-l s$ CIRCUIT}

Fig. 4 shows the layout view of the proposed up level shifter, which has been designed exploiting only metal 1 and 2 wire layers. In $65 \mathrm{~nm}$ CMOS technology, the physical design of $r s c-l s$ occupies a silicon area of only $54.9 \mu \mathrm{m}^{2}(5.04 \mu \mathrm{m} \times$ $10.9 \mu \mathrm{m})$.

To further extend our analysis, we evaluated $r s c-l s$ for the input range $0.45-0.9 \mathrm{~V}$, while keeping the same operating conditions and a $45 \mathrm{fF}$ capacitive load. As is shown in Fig. 5, the proposed up converter provides a good solution across a wide range of input voltages. As shown in Fig. 6, the circuit has a very small delay-energy product across a range of capacitive loads.

To investigate the robustness of the proposed up level shifter against device mismatch, we have performed a 500-points Monte Carlo (MC) simulation. The related results are shown in Fig. 7. The mean, and standard deviation for the delay-energy product are $184.2 \mathrm{e}-24$, and $64.3 \mathrm{e}-24$, respectively.

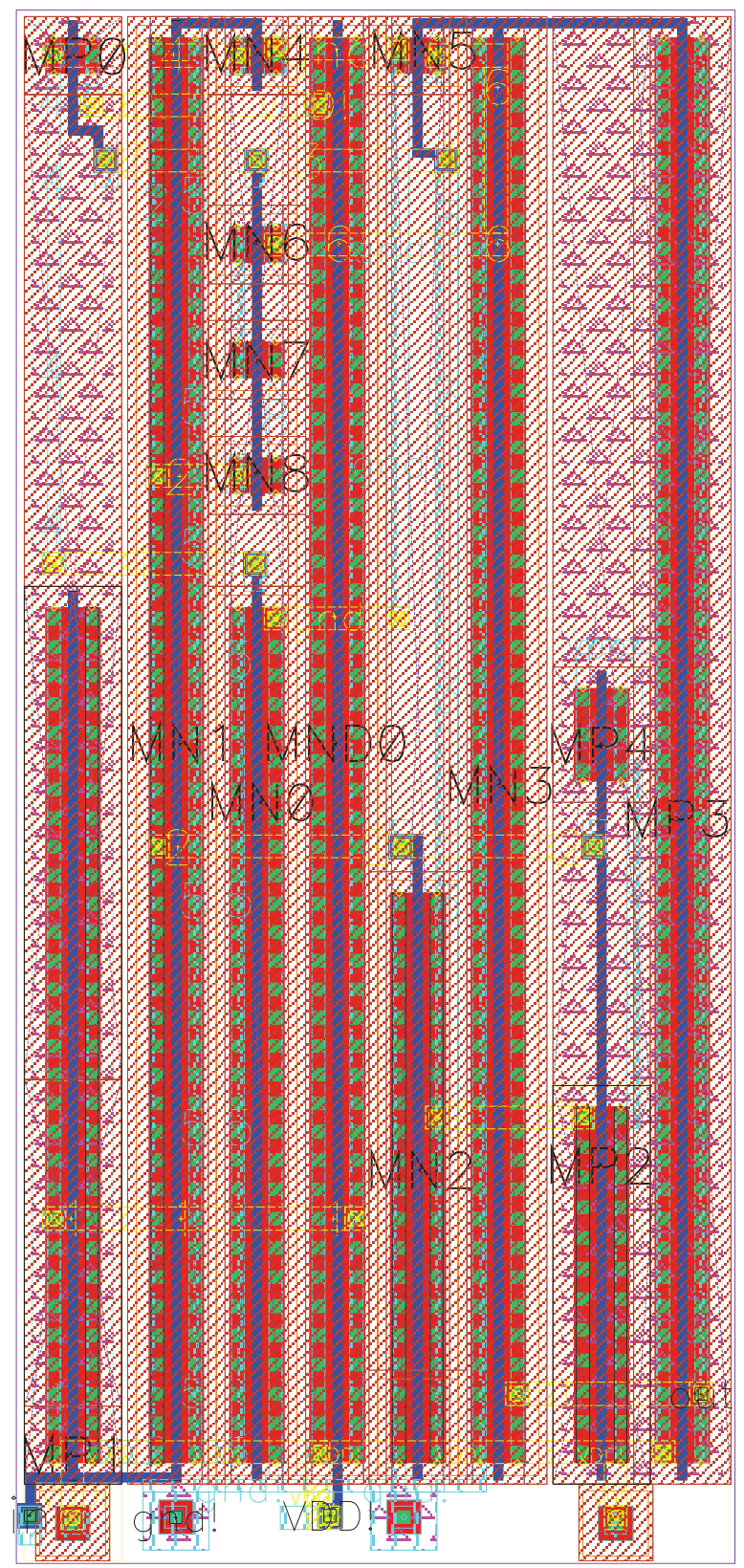

Fig. 4. Layout for $r s c-l s$

\section{COMPARISOn Results}

Table II presents the results obtained for previously reported designs, and our proposed circuit. Design in [2] is implemented with high voltage CMOS transistors due to its greater output voltage. The area for $r s c-l s$ is the lowest among all the designs. The delay and delay-energy product for the proposed $r s c-l s$ circuit are the lowest, with the input signal ranging from 0.45 up to $1 \mathrm{~V}$ and a load of $45 \mathrm{fF}$ and 1 buffer. Comparison of various circuits in Table II shows that [2] can operate under the highest loading condition. The slowest input voltage range is for [5]. 
TABLE II

Comparison of the Proposed Design with Other Up LeVel ShifTers

\begin{tabular}{||c|c|c|c|c|c|c|c||}
\hline Desing & {$[1]$} & {$[2]$} & {$[3]$} & {$[4]$} & {$[5]$} & {$[6]$} & This work \\
\hline Year & 2016 & 2015 & 2014 & 2014 & 2014 & 2014 & 2016 \\
\hline Conversion & Up & Up & Up & Up & Up/Down & Up & Up \\
\hline Technology & $65 \mathrm{~nm}$ & $0.5 \mu \mathrm{m}$ & $90 \mathrm{~nm}$ & $65 \mathrm{~nm}$ & $65 \mathrm{~nm}$ & $65 \mathrm{~nm}$ & $65 \mathrm{~nm}$ \\
& CMOS & HVCMOS & CMOS & CMOS & CMOS & CMOS & CMOS \\
\hline Results & Measured & Measured & Post-layout & Measured & Measured & Measured & Post-layout \\
\hline Area & $31.3 \mu \mathrm{m}^{2}$ & $1.1 \mathrm{~mm}^{2}$ & Not available & Not available & $16.8 \mu \mathrm{m}^{2}$ & $7.8 \mu \mathrm{m}^{2}$ & $3.9 \mu \mathrm{m}^{2}$ \\
\hline Delay & $13.7 \mathrm{~ns}$ & $0.5 \mathrm{~ns}$ & $2 \mathrm{~ns}$ & Not available & Not available & $66 \mathrm{~ns}$ & $0.39 \mathrm{~ns}$ \\
\hline Power & $0.2 \mu \mathrm{W}$ & Not available & Not available & Not available & $1-10 \mathrm{nW}$ & $2 \mu \mathrm{W}$ & $0.21 \mathrm{~mW}$ \\
\hline Frequency & $1 \mathrm{MHz}$ & $3.3 \mathrm{MHz}$ & $1 \mathrm{MHz}$ & Not available & $20 \mathrm{KHz}$ & $72 \mathrm{MHz}$ & $500 \mathrm{MHz}$ \\
\hline EDP $(\mathrm{J} \times \mathrm{s})$ & $1245.33 \mathrm{e}-24$ & Not available & $2900 \mathrm{e}-24$ & Not available & Not available & $1848 \mathrm{e}-24$ & $174.3 \mathrm{e}-24$ \\
\hline Load & $100 \mathrm{fF}$ & $500 \mathrm{pF}$ & $100 \mathrm{fF}$ & Not available & Not available & $1 \mathrm{buffer}$ & $45 \mathrm{fF}+1$ buffer \\
\hline Voltages $(\mathrm{V})$ & 0.1 to 1.2 & 0.5 to 100 & 0.15 to 1 & 0.1 to 2 & 0.1 to 1.2 & 0.12 to 1.2 & 0.45 to 1 \\
\hline
\end{tabular}

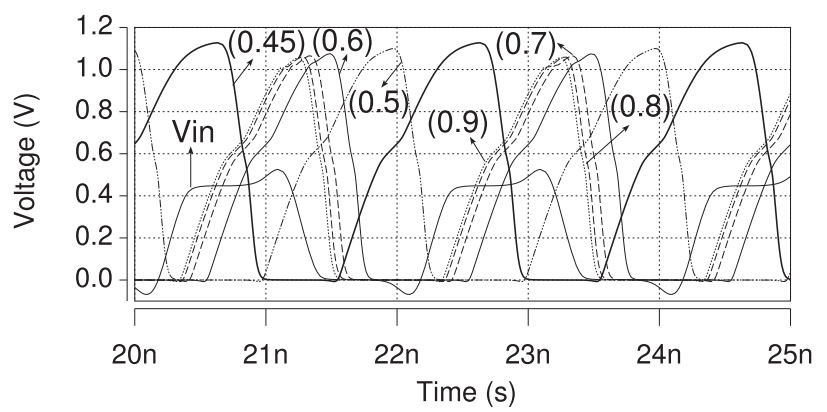

Fig. 5. Waveforms for the post-layout simulation for $r s c-l s$ at $20 \mathrm{fF}$

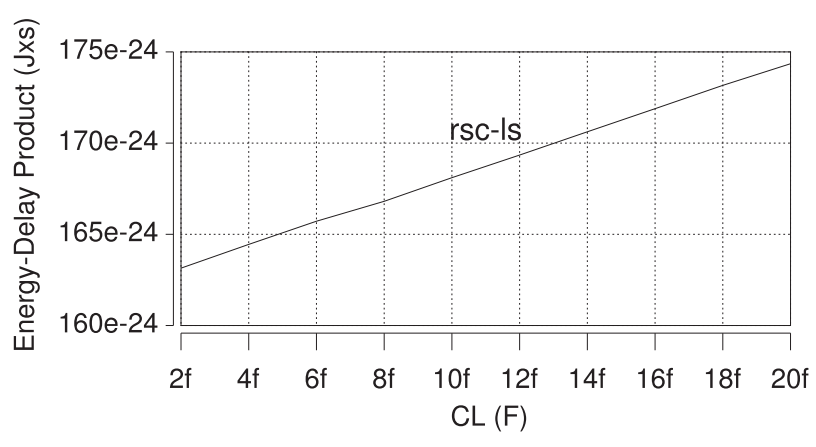

Fig. 6. Delay-energy product for the post-layout simulation for $r s c-l s$

\section{CONCLUSION}

This paper compares the proposed up level shifter with a similar circuit. Both up converters are simulated using a $65 \mathrm{~nm}$ CMOS process. Our design ( $r s c-l s)$ exhibits low static current consumption with lower delay (69\%), lower energy (63\%), and better delay-energy product $(88 \%)$ than circuit used as reference $(b m-l s)$. This is obtained while consuming a 110 fold lower static current, and occupying a 78-fold lower active area than $b m-l s$. From post-layout results, $r s c-l s$ extends

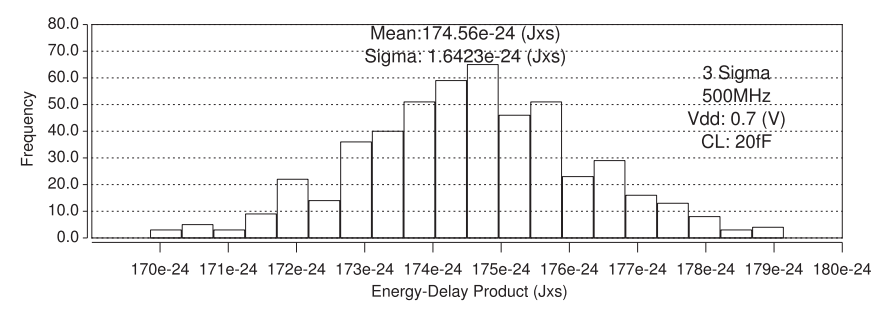

Fig. 7. Histogram for $r s c-l s$

the conversion range down to $0.45 \mathrm{~V}$, with a silicon area of $54.9 \mu \mathrm{m}^{2}(5.04 \mu \mathrm{m} \times 10.9 \mu \mathrm{m})$. Moreover, when converting a $0.7 \mathrm{~V}$ input to $1 \mathrm{~V}$ output at $20 \mathrm{fF}$ it exhibits propagation delay of $0.39 \mathrm{~ns}$ and energy of consumption of $0.43 \mathrm{pJ}$.

\section{ACKNOWLEDGMENT}

This work was funded by project SURF (TEC2014-60527C2-1-R) of the Spanish Ministry of Economy and Competitiveness.

\section{REFERENCES}

[1] L. Wen, X. Cheng, S. Tian, H. Wen, and X. Zeng, Subthreshold Level Shifters With Self-Controlled Current Limiter by Detecting Output Error, IEEE Tran. on Cir. and Sys., II: Exp. Bri., vol. 63, no. 4, pp. 346-350, Apr. 2016.

[2] Z. Liu, L. Cong, and H. Lee, Design of On-Chip Gate Drivers with Power-Efficient High-Speed Level-Shifting and Dynamic Timing Control for High-Voltage Synchronous Switching, 2014 IEEE Jou. of Sol.-Sta. Cir., vol. 50, no. 6, pp. 1463-1477, Jun. 2015.

[3] C.-R. Huang, and L.-Y. Chiou, A Limited-Contention Cross-Coupled Level Shifter for Energy-Efficient Subthreshold-to-Superthreshold Voltage Conversion, 2014 Int. Soc Des. Con., (ISOCC), pp. 142-143, Nov. 2014.

[4] M.-F. Chang, C.-W. Wu, J.-Y. Hung, Y.-C. King, C.-J. Lin, M.S. Ho, C.-C. Kuo, and S.-S. Sheu, A Low-Power Subthreshold-toSuperthreshold Level-Shifter for Sub-0.5V Embedded Resistive RAM (ReRAM) Macro in Ultra Low-Voltage Chips, 2014 IEEE Asi. Pac. Con. on Cir. and Sys., (APCCAS), pp. 695-698, Nov. 2014.

[5] S.-C. Luo, C.-J. Huang, and Y.-H. Chu, A Wide-Range Level Shifter Using a Modified Wilson Current Mirror Hybrid Buffer, IEEE Tran. on Cir. and Sys., I: Reg. Pap., vol. 61, no. 6, pp. 1656-1665, Jun. 2014.

[6] B. Mohammadi, and J. N. Rodrigues, A 65nm Single Stage 28fJ/cycle 0.12 to $1.2 \mathrm{~V}$ Level-Shifter, 2014 IEEE Int. Sym. on Cir. and Sys., (ISCAS), pp. 990-993, Jun. 2014. 